\title{
_Conference Diary
}

\author{
APRIL 1994 \\ 24-27 11th Industrial Minerals International Congress, Berlin, Germany. Details: \\ Conference Department, Metall Bulletin, Park House, 3 Park Terrace, Worcester \\ Park, Surrey KT4 7HY, England. Tel. (081)-330-4311, Fax: (081)-337-8943.
}

\section{MAY 1994}

10-20 International Symposium "The Problems of Complex Ores Utilization (Cu, $\mathrm{Ni}, \mathrm{Co}, \mathrm{Sn}, \mathrm{Al}, \mathrm{Mg}$, Ti and Noble Metals), Saint Petersburg, Russia. Details: Dr. I.N. Beloglazov, PNIL KIR, Saint Petersburg Mining Institute, 21 Line, 2, St. Petersburg, 199026 Russia. Tel. (812) 2188660, Fax: (812) 2185463.

17-19 8th European Symposium COMMINUTION, Stockholm, Sweden. Details: Prof. E. Forssberg, Division of Mineral Processing, Lulea University of Technology, S-951 87 Lulea, Sweden. Tel.: (46) 920-913 11, Fax.: (46) 92097364.

\section{JUNE 1994}

06-08 Innovations in Mineral Processing, Sudbury, Canada. Details: Dr. T. Yalcin, Laurentian University, Sudbury, Ontario, P3E 2C6, Canada. Tel. (705)-675-1151, Fax. (705)-675-4862.

06-10 International Conference of Powder Metallurgy, Paris, France. Details: SF 2M, Immeuble Elysees La Defence, Cedex 35, F-92072 Paris La Defence, France. Tel.: (1)-47678871, Fax: (1)-47678577.

14-18 Second European Metals Conference, Saxony, Germany. Details: GDMP, P.O. Box 1054, D-3392 Clausthal-Zellerfeld, Germany.

15-17 International Conference on Nitromagnetics, Honolulu, Hawaii, USA. Details: Mr. T. Kurino, The Society of Non-Traditional Technology, Tokyo, Japan. Tel.: 8133503 4681, Fax: 81335970535.

20-23 6th Joint MMM-INTERMAG Conference, Albuquerque, New Mexico, U.S.A. 
21st Century Ferrous Metallurgy in Russia and CIS, Moscow, Russia. Details: I.P. Bardin Central Research Institute of Iron and Steel Industry, 9/23 2-ya Baumanskaya, Moscow 107005, Russia. Fax: 261-78-73, Telex: 411580.

\section{JULY 1994}

3-6 6th AusIMM Extractive Metallurgy Conference, Brisbane, Queensland, Australia. Details: Conference Secretary, Department of Mining and Metallurgical Engineering, The University of Queensland, St. Lucia, Qld 4072, Australia. Tel. (7) 3653738 , Fax: (7) 3653888.

\section{AUGUST 1994}

4-5 13th Annual Mineral Processing Symposium, Gordon's Bay, Cape Province, South Africa. Details: Mrs. M. Winter, Dept. of Chemical Engineering, University of Cape Town, South Africa. Tel. 021-6502518, Fax: 021-6503775.

\section{SEPTEMBER 1994}

4-8 XV Congress of the Council of Mining and Metallurgical Institutions, Sun City, Bophuthatswana, South Africa. Details: Bill Emmett, Congress Manager 15th CMMI Congress, P.O. Box 809, Johannesburg 2000, South Africa. Tel. 27-11-838-8211, Fax: 27-11-834-1884.

6-8 5th International Mineral Processing Symposium, Cappadocia, Turkey. Details: Prof. H. Demirel, Dept. of Mining Engineering, Hacettepe University, Beytepe-Ankara 06532, Turkey. Tel.: (4) 235-20055, Fax: (4) 235-4314.

11-15 13th International Workshop and 8th Symposium on Rare-Earth Magnets and Their Applications, Birmingham U.K. Details: Prof. I.R. Harris, School of Metallurgy and Materials, The University of Birmingham, Edgbaston, Birmingham B15 2TT. U.K. Fax: 44-214145232.

26-28 Minerals Engineering '94, Lake Tahoe, USA. Details: Camborne School of Mines, Redruth, Cornwall, England. Fax: (209) 716977.

\section{OCTOBER 1994}

25-28 Electromagnetic Processing of Materials, Nagoya, Japan. Details: Secretariat EPM '94, The Iron and Steel Institute of Japan, Tokyo, Tel. (81) 3 3279 6021, Fax: (81) 332451355. 
$\underline{\text { APRIL } 1995}$

18-21 INTERMAG Conference, San Antonio, Texas, U.S.A.

\section{OCTOBER 1995}

23-27 XIX International Mineral Processing Congress, San Francisco, CA, USA. Details: Meetings Department, Society for Mining, Metallurgy and Exploration, P.O. Box 625002, Littleton, Colorado 80162, USA. Tel.: 303-973-9550, Fax: 303-979-3461.

\section{NOVEMBER 1995}

6-9 40th Conference on Magnetism and Magnetic Materials, Philadelphia, PA, U.S.A.

APRIL 1966

INTERMAG Conference, Budapest, Hungary

\section{NOVEMBER 1966}

12-15 41st Conference on Magnetism and Magnetic Materials, Atlanta, Georgia, U.S.A.

\section{SEPTEMBER 1997}

21-26 XX International Mineral Processing Congress, Aachen, Germany. Details: GDMB, P.O. Box 1054, D-38668 Clausthal-Zellerfeld, Tel.: (49) 5353-3438, Fax: (49) 5323-78804. 